\title{
A NEW CHARACTERIZATION OF INFINITE DIMENSIONAL TEICHMÜLLER SPACES
}

\author{
CLIFFORD J. EARLE and ROBERT S. FOWLER
}

\section{Introduction}

Let $\Gamma$ be any Fuchsian group, finitely generated or not, acting freely on the open unit disk $\Delta$. In [3] we showed that the Teichmüller space $T(\Gamma)$ enjoys a universal property for certain "marked families" of Riemann surfaces. That property uniquely characterizes the complex manifold $T(\Gamma)$. Our purpose here is to give a somewhat simpler characterization of $T(\Gamma)$ by combining our results from [3] with two other ingredients. One is the $\lambda$-lemma of Mañé, Sad, and Sullivan [7]. The other is a recent theorem of Tukia [8], which asserts that $T(\Gamma)$ is contractible. For a short proof of Tukia's theorem, see [2].

Our characterization of $T(\Gamma)$ is given by the theorem in Section 3. This theorem, which was already known for finite dimensional spaces $T(\Gamma)$ (see especially Theorem 4.1 of Grothendieck [4]), provides the first characterization of the infinite dimensional Teichmüller spaces that makes no mention of quasiconformal or quasisymmetric mappings. Of course this does not eliminate quasiconformal mappings from the theory. On the contrary, their role is as important as ever, but now, thanks to the $\lambda$-lemma, they enter as an inevitable consequence of other natural assumptions.

\section{Simple holomorphic families}

Our characterization of $T(\Gamma)$ is in terms of certain special families of Riemann surfaces, which we call simple holomorphic families. By definition, a simple holomorphic family consists of a pair of connected complex manifolds $E$ and $B$, with $B$ simply connected, and a surjective holomorphic map $\pi: E \rightarrow B$ satisfying two additional conditions. First, $\pi$ is required to have "horizontally holomorphic" local trivializations (cf. [6, pp. 15 and 16]). This means there are open sets $B_{\alpha}$ that cover $B$, a space $X$, and homeomorphisms $\theta_{\alpha}$ (the local trivializations) of $B_{\alpha} \times X$ onto $\pi^{-1}\left(B_{\alpha}\right)$ such that $\pi\left(\theta_{\alpha}(t, x)\right)=t$ for all $(t, x) \in B_{\alpha} \times X$ and

(2.1) for each $x$ in $X$, the map $t \mapsto \theta_{\alpha}(t, x)$ from $B_{\alpha}$ into $E$ is holomorphic.

This research was partly supported by the National Science Foundation. 
The maps $t \mapsto \theta_{\alpha}(t, x)$ provide local holomorphic sections of $\pi$ through any point of $E$, so the implicit function theorem implies that each fiber $\pi^{-1}(t), t \in B$, is a closed complex submanifold of $E$. Our second and last condition on $\pi$ is that each fiber $\pi^{-1}(t)$ should be a Riemann surface.

We call attention to the fact that we allow the complex manifolds $E$ and $B$ to be infinite dimensional, modelled on complex Banach spaces. A holomorphic map is a Fréchet differentiable map whose Fréchet derivative is complex linear at every point.

By definition, a map of holomorphic families from the simple family $\pi^{\prime}: E^{\prime} \rightarrow B^{\prime}$ to the simple family $\pi: E \rightarrow B$ is a pair of holomorphic maps $f: B^{\prime} \rightarrow B$ and $g: E^{\prime} \rightarrow E$ such that $g$ restricts to a bijective map from the fiber $\left(\pi^{\prime}\right)^{-1}(t)$ to the fiber $\pi^{-1}(f(t))$ for each $t \in B^{\prime}$. A map of holomorphic families is an isomorphism if $f$ and $g$ are biholomorphic. An isomorphism is an automorphism if $B^{\prime}=B$ and $E^{\prime}=E$.

\section{Statement of the theorem}

Our theorem characterizes $T(\Gamma)$ as the base space of a simple holomorphic family with special properties.

Theorem. Let $\Gamma$ be a Fuchsian group acting freely on the open unit disk $\Delta$, and let $X=\Delta / \Gamma$. There is a simple holomorphic family $\pi: E \rightarrow B$ with these properties:

(3.1) if $\pi^{\prime}: E^{\prime} \rightarrow B^{\prime}$ is a simple holomorphic family with some fiber $\left(\pi^{\prime}\right)^{-1}(t)$ biholomorphically equivalent to $X$, there is a map of holomorphic families $(f, g)$ from

$$
\pi^{\prime}: E^{\prime} \rightarrow B^{\prime} \text { to } \pi: E \rightarrow B
$$

(3.2) the above map $(f, g)$ is unique up to an automorphism of $\pi: E \rightarrow B$.

These properties determine $\pi: E \rightarrow B$ up to isomorphism, and $B$ is biholomorphically equivalent to the Teichmüller space $T(\Gamma)$.

Property (3.2) means that if $\left(f_{1}, g_{1}\right)$ and $\left(f_{2}, g_{2}\right)$ are two maps of holomorphic families from $\pi^{\prime}: E^{\prime} \rightarrow B^{\prime}$ to $\pi: E \rightarrow B$, there is an automorphism $(\varphi, \psi)$ of $\pi: E \rightarrow B$ so that $f_{2}=\varphi \circ f_{1}$ and $g_{2}=\psi \circ g_{1}$.

\section{The uniqueness proof}

We shall prove here that (3.1) and (3.2) determine $\pi: E \rightarrow B$ up to isomorphism. First we show that $\pi: E \rightarrow B$ has a fiber biholomorphically equivalent to $X$. This follows from (3.1) because the projection $\pi_{1}: \Delta \times X \rightarrow \Delta$ defines a simple holomorphic family all of whose fibers are $X$. If $f: \Delta \rightarrow B$ and $g: \Delta \times X \rightarrow E$ define a map of holomorphic families, then $\pi^{-1}(f(z))$ is equivalent to $X$ for every $z$ in $\Delta$. 
Now suppose $\pi: E \rightarrow B$ and $\pi^{\prime}: E^{\prime} \rightarrow B^{\prime}$ both have properties (3.1) and (3.2). Both have a fiber equivalent to $X$, so (3.1) gives maps of holomorphic families $(f, g)$ and $\left(f^{\prime}, g^{\prime}\right)$ from $\pi: E \rightarrow B$ to $\pi^{\prime}: E^{\prime} \rightarrow B^{\prime}$ and from $\pi^{\prime}: E^{\prime} \rightarrow B^{\prime}$ to $\pi: E \rightarrow B$ respectively. The pairs $\left(f^{\prime} \circ f, g^{\prime} \circ g\right)$ and (id, id) both are maps of holomorphic families from $\pi: E \rightarrow B$ to itself, so (3.2) says that $\left(f^{\prime} \circ f, g^{\prime} \circ g\right)=(\varphi, \psi)$ is an automorphism of $\pi: E \rightarrow B$. Similarly $\left(f \circ f^{\prime}, g \circ g^{\prime}\right)=\left(\varphi^{\prime}, \psi^{\prime}\right)$ is an automorphism of $\pi^{\prime}: E^{\prime} \rightarrow B^{\prime}$. It follows easily that $f$ and $g$ are biholomorphic maps, as required.

\section{Proof of a lemma}

Our proof that there is a simple family with properties (3.1) and (3.2) hinges on the following lemma, which is a slight generalization of the $\lambda$-lemma. This is where quasiconformal mappings enter the picture.

Lemma. Let $\theta_{\alpha}: B_{\alpha} \times X \rightarrow \pi^{-1}\left(B_{\alpha}\right)$ be a local trivialization of $\pi: E \rightarrow B$ defined over the connected open set $B_{\alpha} \subset B$. If $\theta_{\alpha}$ satisfies (2.1), then the map $\theta_{\alpha}(t, x) \mapsto \theta_{\alpha}(s, x)$ from $\pi^{-1}(t)$ to $\pi^{-1}(s)$ is quasiconformal for any fixed $t$ and $s$ in $B_{\alpha}$.

Proof. It will be helpful to introduce some notation. We write $X_{t}=\pi^{-1}(t)$ for $t \in B_{\alpha}$, and we define the homeomorphism $\varphi_{t}: X \rightarrow X_{t}$ by $\varphi_{t}(x)=\theta_{\alpha}(t, x)$. The lemma asserts that $\psi_{s t}=\varphi_{s} \circ \varphi_{t}^{-1}$ is quasiconformal.

Since $B_{\alpha}$ is connected, it suffices to prove that each $t \in B_{\alpha}$ has a neighborhood $U$ such that $\psi_{s t}$ is quasiconformal for all $s \in U$. We may therefore assume that $B_{\alpha}$ is an open coordinate ball centered at some point $t_{0}$. Since any point of $B_{\alpha}$ belongs to a one-dimensional disk through $t_{0}$, we may and do assume that $B_{\alpha}$ is the open unit disk $\Delta$. Since we may replace $\theta_{\alpha}(t, x)$ by $\theta_{\alpha}\left(t, \varphi_{0}^{-1}(x)\right)$, we shall also assume that $X$ is the Riemann surface $X_{0}$ and that $\theta_{\alpha}(0, x)=\varphi_{0}(x)=x$ for all $x \in X$.

Let $U \subset X$ be any relatively compact coordinate disk, and let $U_{t}=\varphi_{t}(U) \subset X_{t}$. Our first step is to prove that $\psi_{s t}: U_{t} \rightarrow U_{s}$ is quasiconformal for all $s, t \in \Delta$. Again this need be proved only for $s$ near a given $t$. Let $t \in \Delta$ be fixed. By compactness, there are open sets $V_{1}, \ldots, V_{n} \subset U$ and $D \subset \Delta$ so that $D$ is a disk that contains $t$, the $V_{j}$ cover $U$, and $E$ has local coordinates $(s, \zeta)$, defined on $\theta_{\alpha}\left(D \times V_{j}\right)$, in terms of which

$$
\theta_{\alpha}(s, z)=\left(s, f_{j}(s, z)\right) \text { for }(s, z) \in D \times V_{j}, \quad 1 \leqq j \leqq n .
$$

Here $f_{j}$ maps $D \times V_{j}$ into $C$, and $\varphi_{s}(z)=f_{j}(s, z)$ if $z \in V_{j}$.

We shall complete the proof of Step one by showing that $\psi_{s t}$ is quasiconformal in each set $\varphi_{t}\left(V_{j}\right) \subset U_{t}$. That follows directly from the $\lambda$-lemma. Indeed, using local coordinates we have a map $(s, \zeta) \mapsto \psi_{s t}(\zeta)$ from $D \times\left\{f(t, z) ; z \in V_{j}\right\}$ into $C$. This map is holomorphic in $s$ for fixed $\zeta$, injective in $\zeta$ for fixed $s$, and is the identity in $\zeta$ when $s=t$, so it is quasiconformal in $\zeta$ for each fixed $s$, by the $\lambda$-lemma [7]. Step one is proved. 
The second and last step is to prove that the map $\psi_{s t}: U_{t} \rightarrow U_{s}$ is in fact $K$-quasiconformal for a number $K$ that depends only on $t$ and $s$, not on the coordinate disk $U$. Since $\varphi_{0}$ is the identity, Step one implies that each map $\varphi_{t}$ is quasiconformal. Let

$$
\mu_{t}(z)=\frac{\left(\varphi_{t}\right)_{\bar{z}}(z)}{\left(\varphi_{t}\right)_{z}(z)} .
$$

Since $\theta_{\alpha}(t, z)$ depends holomorphically on $t$ for fixed $z$, Lemma 9 of [3] implies that $t \mapsto \mu_{t}$ is a holomorphic map from $\Delta$ into $L^{\infty}(U, C)$. Now $\left\|\mu_{t}\right\|<1$ for all $t \in \Delta$, and $\mu_{0}(z) \equiv 0$ because $\varphi_{0}$ is the identity, so Schwarz's lemma [5, Theorem 3.13.4] gives $\left\|\mu_{t}\right\| \leqq|t|$ for all $t \in \Delta$. Therefore $\varphi_{t}$ is $K_{t}$-quasiconformal, with

$$
K_{t}=\frac{1+|t|}{1-|t|},
$$

and $\psi_{s t}$ is $K_{s} K_{t}$-quasiconformal. The lemma is proved.

\section{The existence proof}

Let $T(\Gamma)$ be the Teichmüller space of the Fuchsian group $\Gamma$. By results of Bers [1], $\Gamma$ acts on the Bers fiber space $F(\Gamma)$, producing a complex manifold $V(\Gamma)=$ $F(\Gamma) / \Gamma$ and a holomorphic map $\pi: V(\Gamma) \rightarrow T(\Gamma)$. By Theorem 1 of [3], $\pi$ has local trivializations $\theta_{\alpha}$ that satisfy (2.1) with $X=\Delta / \Gamma$. By Tukia's theorem [8], $T(\Gamma)$ is contractible, hence simply connected, so $\pi: V(\Gamma) \rightarrow T(\Gamma)$ is a simple holomorphic family. We claim that it has properties (3.1) and (3.2). Property (3.2) is a special case of Corollary 1 of Theorem 2 in $[3, \S 7.7]$, so it remains to prove (3.1).

Let $\pi^{\prime}: E^{\prime} \rightarrow B^{\prime}$ be a simple holomorphic family with some fiber biholomorphically equivalent to $X=\Delta / \Gamma$. By definition, a strong local trivialization over the open set $U \subset B^{\prime}$ is a homeomorphism $\theta$ of $U \times X^{\prime}$ onto $\left(\pi^{\prime}\right)^{-1}(U)$ such that $\pi(\theta(t, x))=t, X^{\prime}=\Delta / \Gamma^{\prime}$ for some Fuchsian group $\Gamma^{\prime}$ acting freely on $\Delta$, the map $\theta(\cdot, x)$ is holomorphic for each fixed $x$, and the map $\theta(t, \cdot)$ is quasiconformal for each fixed $t$. The Riemann surface $X^{\prime}$ is called the fiber model of $\theta$.

We claim there is a strong local trivialization $\theta$ with fiber model $X$ defined in a neighborhood of any point $t_{0} \in B^{\prime}$. Indeed, the definition of simple family gives a horizontally holomorphic local trivialization $\theta_{\alpha}$ defined over some connected open neighborhood $B_{\alpha}$ of $t_{0}$. Since all fibers are homeomorphic to $X$, we may assume that the domain of $\theta_{\alpha}$ is $B_{\alpha} \times X$. By the lemma, all fibers are quasiconformally equivalent to each other, hence to $X$, so there is a homeomorphism $f: X \rightarrow X$ such that the map $x \mapsto \theta_{\alpha}\left(t_{0}, f(x)\right)$ from $X$ to $\left(\pi^{\prime}\right)^{-1}\left(t_{0}\right)$ is quasiconformal. It follows at once from the lemma that

$$
\theta(t, x)=\theta_{\alpha}(t, f(x))
$$

is a strong local trivialization defined over a neighborhood of $t_{0}$. 
Since $B^{\prime}$ is simply connected, we can use Theorems 1 and 4 of [3] to obtain a map of holomorphic families from $\pi^{\prime}: E^{\prime} \rightarrow B^{\prime}$ to $\pi: V(\Gamma) \rightarrow T(\Gamma)$. Therefore the simple family $\pi: V(\Gamma) \rightarrow T(\Gamma)$ has property (3.1), and the proof of the theorem is complete.

\section{References}

[1] Bers, L.: Fiber spaces over Teichmüller spaces. - Acta Math. 130, 1973, 89-126.

[2] Douady, A., and C. J. Earle: Conformally natural extension of homeomorphisms of the circle. - To appear.

[3] EARLE, C. J., and R. S. Fowler: Holomorphic families of open Riemann surfaces. - To appear.

[4] Grothendieck, A.: Techniques de construction en géométrie analytique. - Séminaire Henri Cartan, 13e année: 1960/61, Familles d'espaces complexes et fondements de la géométrie analytique, Exp. 7, Paris, 1962, 1-33.

[5] Hille, E., and R. S. Phillips: Functional analysis and semi-groups. - American Mathematical Society Colloquium Publications 31. American Mathematical Society, Providence, R.I., 1957.

[6] Hubbard, J. H.: Sur les sections analytiques de la courbe universelle de Teichmüller. - Mem. Amer. Math. Soc. 4:166, 1976, 1-137.

[7] MaÑÉ, R., P. SAD, and D. Sullivan: On the dynamics of rational maps. - To appear.

[8] Tukia, P.: To appear.

Cornell University

Department of Mathematics

Ithaca, New York 14853

USA

Received 4 November 1983
Pennsylvania State University

Department of Mathematics

Media, Pennsylvania 19063

USA 